\title{
A Versatile Biomolecular Charge-Based Sensor Using Oxide-Gated Carbon Nanotube Transistor Arrays
}

\author{
Herman Pandana, Konrad H. Aschenbach, Daniel R. Lenski, Michael S. Fuhrer, Javed Khan, and \\ Romel D. Gomez, Senior Member, IEEE
}

\begin{abstract}
Label-free deoxyribonucleic acid (DNA) hybridization detection using carbon nanotube transistor (CNT) arrays is demonstrated. The present scheme is distinguished from other CNT sensing methods as it uses a gate oxide overlayer on top of the carbon nanotubes, which function solely as charge sensors but are not participants in the chemical binding process. Because it involves DNA probe attachment on the gate oxide rather than on the CNT, this approach allows the use of conventional DNA functionalization and bioassay protocols, and is less prone to false positives. The signal sought is a few tens of millivolts in threshold voltage shift due to the increase of surface charges after target hybridization. The hybridization detection is shown to be highly specific and sensitive to a minimum concentration of about $30 \mathrm{nM}$ of 61-mer DNA. Despite differences in the transistor properties due to the spread in the CNT parameters during fabrication, the yields are very high and the sensing characteristics are uniformly consistent in nearly all transistors.
\end{abstract}

Index Terms-Biosensor, carbon nanotube, label-free deoxyribonucleic acid (DNA) detection, transistor.

\section{INTRODUCTION}

D EOXYRIBONUCLEIC ACID (DNA) microarrays are indispensable tools in molecular biology to investigate gene expression at the transcription level and to identify transcription factors. In a conventional DNA microarray experiment, an array of known single stranded DNA sequences, called probes, are immobilized on a substrate and later exposed to an unknown set of target genes or DNA that have been chemically tagged with reporter molecules. In places on the array where the probe and target sequences are complementary, Watson-Crick hybridization occurs and the locations of these specific binding events are revealed by the reporter molecules. Current DNA microarrays rely on fluorescent detection, and require inevitable complex protocols such as enzymatic amplification of DNA sam-

Manuscript received August 3, 2007; revised October 26, 2007; accepted January 15,2008 . This work was supported in part by the National Institute of Health under SAIC Grant 0701097058, and in part by the Laboratory for Physical Sciences under Grant H98230-04-C-0470. The associate editor coordinating the review of this paper and approving it for publication was Dr. James Jensen.

H. Pandana, K. H. Aschenbach, and R. D. Gomez are with the Department of Electrical and Computer Engineering, University of Maryland, College Park, MD 20742 USA (e-mail: hpandana@glue.umd.edu; konrad@umd.edu; rdgomez@ece.umd.edu).

D. R. Lenski and M. S. Fuhrer are with the Department of Physics and Center for Nanophysics and Advanced Materials, University of Maryland, College Park, MD 20742 USA (e-mail: lenski@umd.edu; mfuhrer@umd.edu).

J. Khan is with the Pediatric Oncology Branch, National Cancer Institute, National Institutes of Health, Gaithersburg, MD 20877 USA (e-mail: khanjav@mail.nih.gov).

Color versions of one or more of the figures in this paper are available online at http://ieeexplore.ieee.org.

Digital Object Identifier 10.1109/JSEN.2008.922724 ples, fluorescent labeling of targets, followed by detection by optical scanners. It is, therefore, desirable to have a means for label-free DNA hybridization detection to bypass some, if not all of these protocols. One natural way is to detect the inherent electric charges on the phosphate backbone of DNA, which is ionized in buffer solution.

The idea of charge-based detection has been around since the ion-sensitive field effect transistor (ISFET) was introduced by Bergveld [1] in 1972. The field effect detection usually utilizes an electrolyte-insulator-silicon (EIS) structure. It is basically a metal-oxide-semiconductor (MOS) structure without the metal gate. Charges adsorbed on the EIS structure produce a field effect to shift the flat band potential and/or change the capacitive/resistive impedance across the EIS structure. There has been a lot of work accomplished along this line. The first label-free homo-oligomer DNA detection was demonstrated by Souteyrand et al. [2], who observed a shift in the flat-band potential of the underlying semiconductor in response to the increase of surface charges induced by hybridization between the complementary homo-oligomer strands. Works by others [3], [4] followed, and other forms of transistors such as a MOS structure on cantilevers [5], polysilicon transistors [6], nanowire transistors [7], carbon nanotube transistors (CNTs) [8] were utilized.

In this work, we utilized carbon nanotube field effect transistors (CNTFET) to detect the increase in surface charge upon target DNA hybridization. This approach is similar to that of Star et al. [8], with the important distinction that the carbon nanotube channel is insulated by an oxide layer and is not directly functionalized by probe-DNA. In this manner, the carbon nanotube channel serves only as a sensitive charge sensor, and does not see the chemistry of DNA immobilization and hybridization. Since all of the chemistry is done on the oxide insulating surface, the protocols are very similar and compatible to those used regularly in conventional bioassays. We believe our approach could reduce nonspecific binding of the target DNA directly to the exposed nanotubes, thereby reducing the rate of false positives.

\section{EXPERIMENTS}

\section{A. Device Fabrication}

Device fabrication starts with growing carbon nanotube mats on iron coated silicon oxide substrates. Iron catalyst was prepared by brief iron evaporation of a submonolayer film onto the silicon oxide substrate in an ultra high vacuum chamber. Carbon nanotube mats were grown using chemical vapor deposition (CVD) technique in a tube furnace [9]. The substrate was heated to $850^{\circ} \mathrm{C}$ in the presence of argon as carrier gas inside the furnace, into which growth gases of methane, ethylene, and 


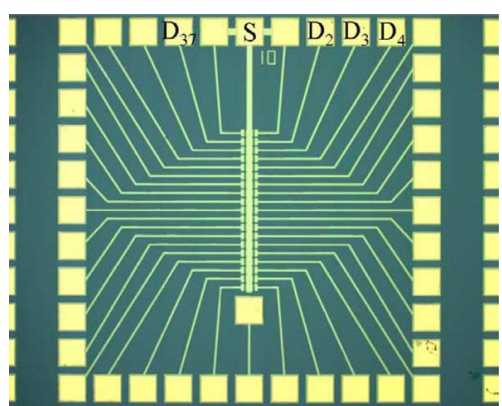

Fig. 1. Layout of the CNTFET array. The center bar denoted by "S" is the common source electrode, shared by other drain electrodes, denoted by "D2," "D3," etc. Each source and drain pair comprise one transistor.

hydrogen were flowed for $10 \mathrm{~min}$. The grown carbon nanotube mats consist of various sizes and chiralities of carbon nanotube, but are mostly single walled, and contain both semiconducting and metallic type in roughly 2:1 proportion.

Source and drain electrodes were defined using standard microlithography. The electrodes consist of a $5 \mathrm{~nm}$ chromium adhesion layer and $25 \mathrm{~nm}$ of aluminum. In order to squeeze many transistors into a small area, we designed a layout (Fig. 1) such that the transistors share one common source electrode. Unwanted nanotubes outside the active area of each transistor were etched away using oxygen plasma. The active area, or the gap between source and drain electrodes where the nanotube channel sits, is $10 \mu \mathrm{m}$ long and $50 \mu \mathrm{m}$ wide. $150 \mathrm{~nm}$ of gold were evaporated on the bonding pad squares for the purpose of subsequent wirebonding in order to mount the chip on a package. The whole chip is passivated with $25 \mathrm{~nm}$ of atomic layer deposited (ALD) aluminum oxide. The bonding pad squares were later opened up for gold wire-bonding to the package.

\section{B. DNA Immobilization and Hybridization}

Probe DNA was immobilized via thiol chemistry onto a silane-functionalized surface. The aluminum oxide surface was functionalized with 3-mercapto-propyl-trimethoxy-silane (MPTMS). First, the aluminum oxide was treated with oxygen plasma to generate a hydroxyl terminated surface, and then exposed to MPTMS vapor. The MPTMS molecules bind covalently to the surface through the hydroxyl groups. $10 \mu \mathrm{M}$ of Acrydite ${ }^{\mathrm{TM}}$-modified probe oligonucleotide, dissolved in TE buffer ( $\mathrm{pH}$ 7), was pipetted to the chip, and was allowed to dry slowly overnight before the unbound mercaptan molecules of the MPTMS were passivated with $50 \mathrm{mM}$ of L-histidine at $\mathrm{pH}$ 7. At this stage, DNA immobilization was complete, and the chip was ready for target hybridization.

Before carrying on with target hybridization, transfer characteristics ( $I_{\mathrm{DS}}-V_{\mathrm{GS}}$ curves) of the transistor array were recorded. The hybridization was done by incubating the target DNA of desired sequence on the chip no longer than $2 \mathrm{~h}$ in $100 \%$ humidity. The sequence of DNA oligonucleotide used in the experiment is summarized in Table I. The target DNA was dissolved in a hybridization buffer, i.e., $10 \mathrm{mM}$ of phosphate buffer saline $\mathrm{pH}$ 7.0, $0.3 \mathrm{M} \mathrm{NaCl}$. The chip was then washed thoroughly in TE buffer, and ready for the transfer characteristic measurement.
TABLE I

DNA Oligonucleotide NAmes AND Sequences

\begin{tabular}{ll}
\hline \multicolumn{1}{c}{ Name } & \multicolumn{1}{c}{ Sequence } \\
\hline probe & 5'-/Acrydite//CAG ATG CCA CAT AAG GGG TTT \\
& GCC ATT TGA TAC CCC TGG GGA ACA TTT \\
& CTG TAA ATA CCA T -3' \\
complementary & 5'-/ATG GTA TTT ACA GAA ATG TTC CCC AGG \\
target & GGT ATC AAA TGG CAA ACC CCT TAT GTG \\
& GCA TCT G -3' \\
non - & 5'-/TTT TTT TTT TTT TTT TTT AGT CCG TGG \\
complementary & TAG GGC AGG TTG GGG TGA CT-3' \\
target & \\
\hline
\end{tabular}

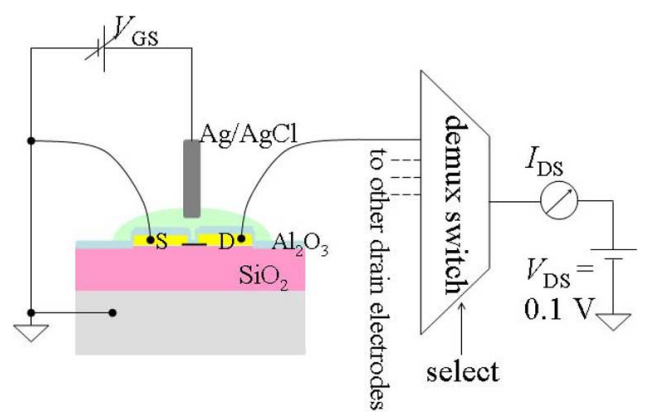

Fig. 2. The schematic of the measurement system. A fixed $100 \mathrm{mV}$ bias was applied between the drain and source electrode. IDS was recorded as VGS was swept to generate transistor transfer characteristic curve. A demux switch, which has an "on" resistance less than $10 \mathrm{~W}$, was used to automate the sequential measurement of all drain currents.

\section{Measurement System}

A schematic of the wiring of the device measurement system is shown in Fig. 2. To measure the top-gate transfer characteristics of the transistors, a constant voltage $V_{\mathrm{DS}}=100 \mathrm{mV}$ was applied to drain and source electrodes, while the current $I_{\mathrm{DS}}$ was read-out. Gate voltage was applied to a $\mathrm{Ag} / \mathrm{AgCl}$ counter electrode in contact with the TE buffer solution during electrolyte gating measurement. The measurement on the transistor array was automated through a demultiplexer-switch to scan every drain electrode in the array.

\section{THEORY}

A thorough theoretical treatment of an EIS structure has been published [10]. We would like to review only several points that are relevant to our work.

First, we need to keep in mind that in our experiment, the oxide surface sees several treatments, from silane functionalization to DNA immobilization and hybridization. We have to be cautious to take into account all surface charges that might be adsorbed on the oxide upon each treatment. In a very simplified model, the threshold voltage shift is given by

$$
\Delta V=\frac{\Delta Q}{C_{\text {eff }}}
$$

where $\Delta Q$ is the added surface charge, and $C_{\text {eff }}$ is the effective capacitance, which is dominated by the double-layer capacitance. This is similar to the expression of the flat-band voltage shift caused by trapped charges in MOS devices. The screening effect by mobile ions in the buffer solution has been taken into 
account by $C_{\text {eff }}$. In a very hand-waving yet elucidating manner, we can express the effective capacitance as [11]

$$
\frac{C_{\text {eff }}}{\text { Area }} \approx \frac{\varepsilon_{\text {buffer }}}{l_{\text {debye }}}=\sqrt{\frac{2 \varepsilon_{\text {buffer }} z^{2} e^{2} c}{k_{B} T}}
$$

where $z$ is the magnitude of the charge of the buffer ions (e.g., $z=1$ for $\mathrm{NaCl}$ ), and $c$ is the buffer concentration; $\epsilon_{\text {buffer }}$ is the buffer dielectric constant, $e$ is electron charge, $k_{B}$ is the Boltzmann constant, and $T$ is temperature. A low ionic strength buffer is desired during measurement to minimize the capacitance in order to yield a larger signal or threshold voltage shift. In the case of DNA hybridization, one should see a positive threshold voltage shift upon target hybridization, since the target DNA carries negative charges on its phosphate backbone. A conflicting requirement on salt concentration during DNA hybridization may complicate or even degrade the detection capability. High salt concentration is required to ensure high yield of hybridization by screening the electrostatic repulsion between the probe-DNA and the target-DNA. Care has to be taken to ensure the reliability of the oxide surface upon exposure to high salt solution during DNA hybridization. If sodium or any other alkali ions used in the hybridization buffer embed themselves into the oxide layer, one would observe a negative threshold voltage shift. Silicon oxide is known to be prone to sodium penetration. Aluminum oxide may provide a better resistance against ion penetration. However, the aluminum oxide was subjected to overnight incubation under salt solution, and eventually yielded to alkali ion penetration as well, as a resulting negative threshold voltage shift was observed. Therefore, we have restricted the hybridization time not to exceed two hours to minimize oxide poisoning.

Second, the hybridization signal is measured through potentiometric measurement of the threshold voltage shift upon hybridization. The magnitude of the signal expected is only a few tens of millivolts, therefore, it is important to take a close look at the voltage drops across the device. The applied voltage is dropped across the nanotube channel, which may be in the form of Schottky barrier or space charge, across the aluminum oxide, across the oxide and electrolyte interface, which varies with amount of surface charges adsorbed, and across the electrolyte and counter electrode interface. It is then crucial to ensure that all voltage drops are well-defined, and not fluctuating or smearing away the signal we are trying to detect. The use of a nonpolarizable counter electrode such as $\mathrm{Ag} / \mathrm{AgCl}$ is required, since if there is a common species on the electrode (i.e., chloride) and in the electrolyte buffer (i.e., chlorine ions in the TE buffer), then the interface potential is well-defined according to the Nernst equation [11]

$$
V_{\mathrm{Ag} / \mathrm{AgCl} \text {-electrolyt e }}=\frac{k_{B} T}{e} \operatorname{In}\left(\frac{c}{c_{\mathrm{PZC}}}\right)
$$

where $c_{\mathrm{PZC}}$ denotes the buffer concentration, or more precisely the chloride ion activity, at the point of zero charge.

\section{RESULTS}

Fig. 3 shows a back-gated $I_{\mathrm{DS}}-V_{\mathrm{BS}}$ transfer characteristic. The voltage was applied to the silicon substrate and the nanotube

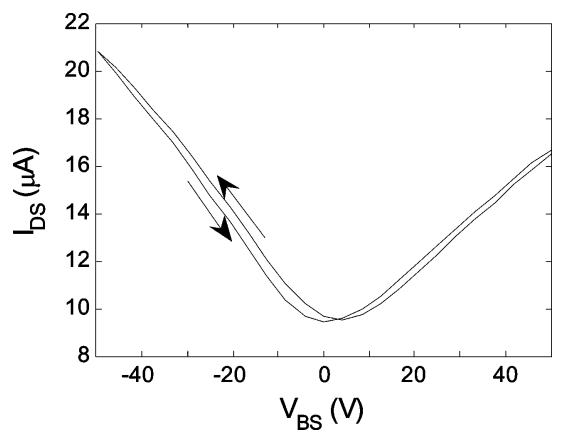

Fig. 3. Back-gated transfer characteristic of $\mathrm{Al}_{2} \mathrm{O}_{3}$ passivated CNTFET transistor shows ambipolar behavior and a small hysteresis.

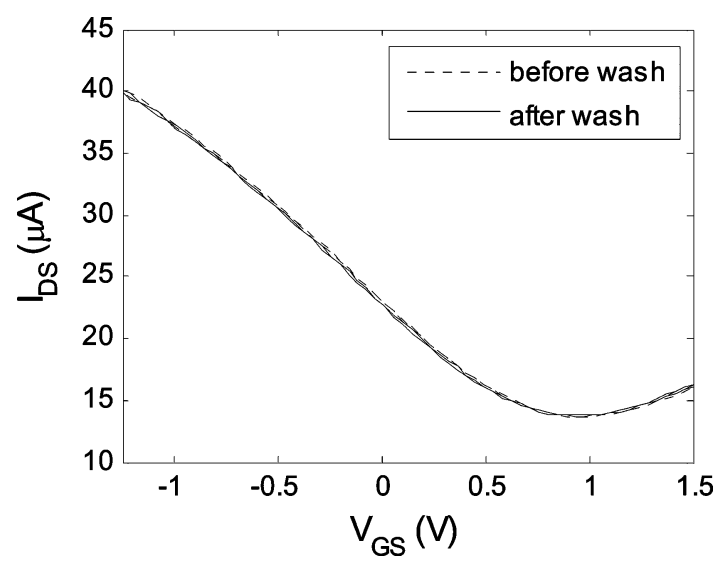

Fig. 4. Top-gated transfer characteristic through $\mathrm{Ag} / \mathrm{AgCl}$ counter electrode and electrolyte buffer. The plot shows very good repeatability of device behavior upon washing, blow-drying, and buffer change.

channel was gated through the 500-nm-thick silicon oxide. The curve, which is representative for top-oxide passivated CNTFET transistors, shows a small hysteresis, and ambipolar behavior of the transistor. This is consistent with the observation of Martel et al. [12] that upon $\mathrm{SiO}_{2}$ passivation and $700{ }^{\circ} \mathrm{C}$ annealing, the originally $p$-type conduction CNTFET device became fully ambipolar. Kim et al. [13] suggested that adsorption of water molecules is responsible for wide hysteresis loop observed in CNTFET. We also observed $p$-type conduction and wide hysteresis loops of $I_{\mathrm{DS}}-V_{\mathrm{BS}}$ curves before passivation. Due to the growth mechanism and film quality of ALD, we think that ALD $\mathrm{Al}_{2} \mathrm{O}_{3}$ passivation of CNTFET achieves both removal of oxygen and water vapor from the carbon nanotubes, thereby resulting in ambipolar behavior and minimal hysteresis in the passivated-CNTFET's transfer characteristics.

Fig. 4 shows a top-gated $I_{\mathrm{DS}}-V_{\mathrm{GS}}$ transfer characteristic. The voltage was applied to the $\mathrm{Ag} / \mathrm{AgCl}$ counter electrode, and the nanotube channel was gated through the 25 -nm-thick aluminum oxide. The plot shows a much-reduced range of voltage needed to gate the channel. It also shows high repeatability upon device washing and change of buffer solution. The ambipolar characteristic is helpful in defining the threshold voltage. We define the threshold voltage as the voltage at which $I_{\mathrm{DS}}$ is minimum. The threshold voltage position was obtained by fitting a parabola around the minimum to the transfer characteristic curve. The shift in threshold voltage, which arises from the additional surface charges, serves as the hybridization signal. 


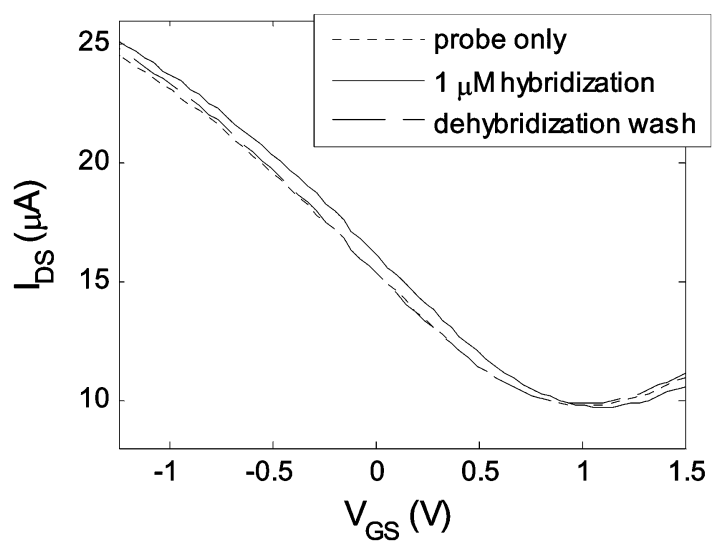

Fig. 5. Transfer characteristic of CNTFET for probe-DNA only, $1 \mathrm{mM}$ complementary target DNA hybridization, and subsequent dehybridization wash to remove the target DNA. The plot shows clear rightward (positive) shift in threshold voltage upon hybridization due to added negative surface charges from the target DNA, and a subsequent leftward shift after target DNA removal.

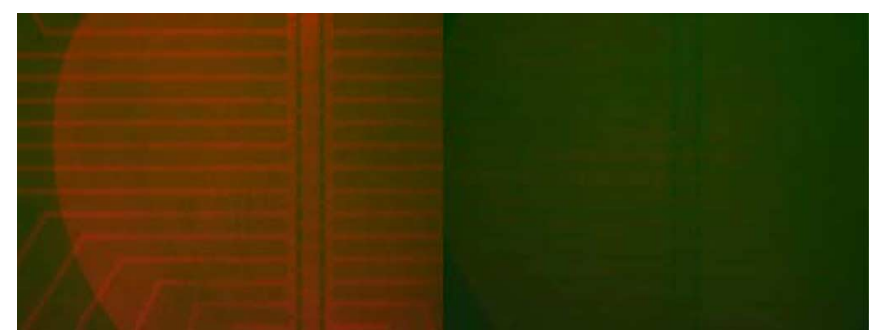

Fig. 6. Fluorescent image taken after transfer characteristic measurement in Fig. 5, (left) fluorescent image of $1 \mathrm{mM}$ complementary target hybridization, (right) fluorescent image after dehybridization wash to remove target DNA.

In order to verify that the threshold shift is indeed due to the target binding, we used fluorescently labeled target DNA to allow simultaneous electrical detection and fluorescent detection of the DNA hybridization. Fig. 5 shows the transistor transfer characteristics for the cases of probe-DNA, target DNA hybridization, and denaturation/dehybridization wash to remove target DNA. The transistors showed a rightward or positive threshold voltage shift upon exposure of $1 \mu \mathrm{M}$ of target DNA hybridization, as expected since more negative charges were adsorbed on the surface. As described in Section III, high salt concentration is required for DNA hybridization and low salt concentration is desired during electrical measurement. Had a significant density of $\mathrm{Na}^{+}$penetrated the oxide layer, we would have observed a left shift in the threshold voltage. To remove the target DNA, the transistors were washed with copious amounts of warm distilled water. After this procedure, which we refer to as dehybridization wash, the threshold voltage reverted back to values before hybridization. The success of hybridization and dehybridization are confirmed by the fluorescence images in Fig. 6, which were taken with the same camera setting (i.e., gain and integration time). We conclude that the target incubation period is long enough to allow for hybridization and short enough to prevent significant oxide poisoning.

To further gain confidence in conclusions, we tested all of the transistors that were available in one array shown in Fig. 1. Fig. 7 summarizes the threshold voltage data of the transistor array

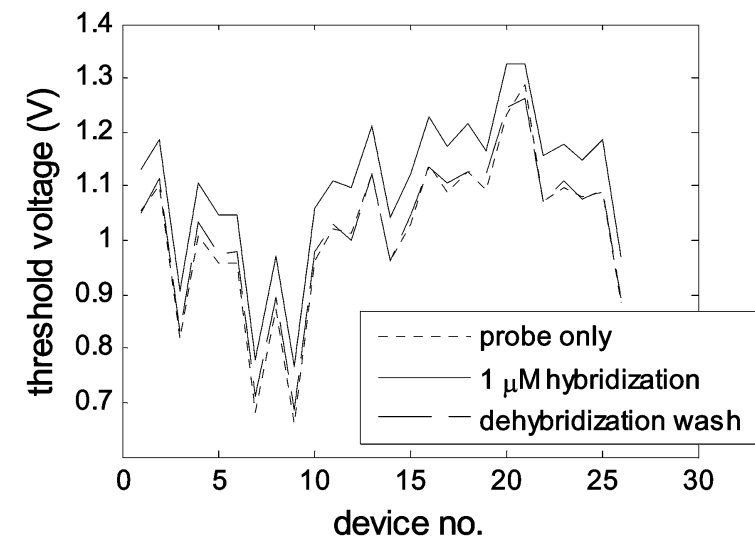

Fig. 7. Threshold voltages of the transistors in the array upon DNA hybridization treatments. The plot shows universal positive shift in threshold voltage of all transistors upon complementary target hybridization, and recovery after target DNA removal through dehybridization wash.

upon DNA treatment. Since carbon nanotube mats were used to fabricate the device, we cannot expect uniformity of threshold voltages across the array. The differences in the initial threshold voltage can be attributed to the uncontrolled variation in each of the transistor channel, such as number of nanotubes crossing the source and drain gap, chirality, and diameter of the nanotubes. However, the plot shows a universal threshold voltage shift of all transistors to the positive (rightward) direction upon DNA hybridization, and universal recovery upon target DNA denaturation. The amount of shift is a function of the amount of added surface charges from the target DNA hybridization, and should not depend on the initial position of the transistor's threshold voltage. The data clearly shows this behavior. More importantly, using a simple model we can estimate how much DNA has bound to the surface. Using $10 \mathrm{mM}$ for the concentration of the TE buffer being used during measurement, (2) gives an areal capacitance of $2.29 \times 10^{-5} \mathrm{~F} / \mathrm{cm}^{2}$. A $60 \mathrm{mV}$ voltage shift for the $1 \mu \mathrm{M}$ target concentration, as shown in Fig. 8, corresponds to an additional surface charge of $1.37 \times 10^{-6} \mathrm{C} / \mathrm{cm}^{2}$, which means that the average surface density of the 61-mer target DNA is $1.4 \times 10^{11}$ molecules $/ \mathrm{cm}^{2}$. Equivalently, the average spacing between target DNA is $27 \mathrm{~nm}$. This number, of course, depends upon many factors including the probe loading and the efficiency of hybridization, and thus, we could expect improvement in sensitivity if the loading and hybridization conditions are optimized.

To address the issue of sensitivity and selectivity of our device, we measured the response at different target concentrations. Fig. 8 shows the amount of threshold voltage shift at $10 \mathrm{nM}, 100 \mathrm{nM}$, and $1 \mu \mathrm{M}$ target DNA concentrations. For this experiment, we used targets that are nonlabeled. We also examined the specificity of the assay by hybridizing $1 \mu \mathrm{M}$ solution, a fairly high concentration, of noncomplementary target DNA. The noncomplementary signal serves as the noise floor and, therefore, we conclude that the sensitivity of our assay is somewhere between 10 to $100 \mathrm{nM}$. This sensitivity is many orders of magnitude lower than the state-of-the-art fluorescent techniques which can detect single atoms. However, to be useful for diagnostic applications, the sensitivity of $1 \mathrm{pM}$ or better is needed. Our system is far from being optimized but there are 


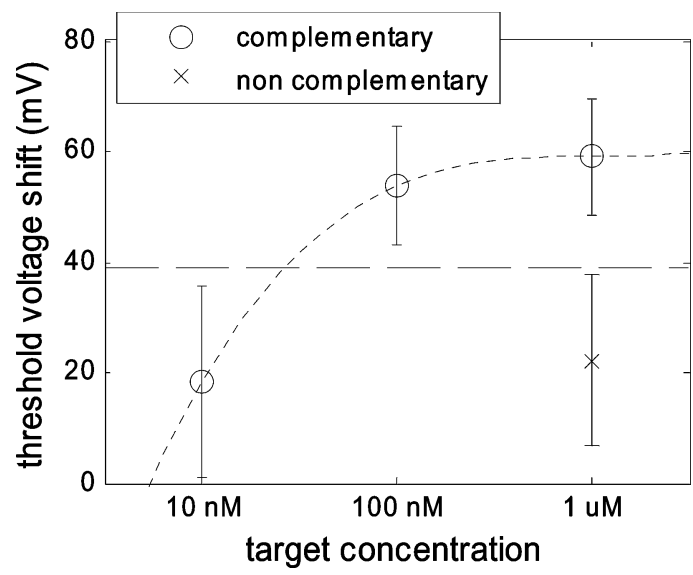

Fig. 8. Titration curve shows sensitivity and specificity of the sensor. The sensitivity level is somewhere between 10 to $100 \mathrm{nM}$. The dotted curve is an interpolation of the data, to show that the sensitivity level is around $30 \mathrm{nM}$. The signal difference between $1 \mathrm{mM}$ hybridization of complementary and that of noncomplementary target clearly shows the specificity of the sensor.

compelling estimates that suggest the feasilibity of achieving this level of detection through improved probe immobilization chemistry, better hybridization protocols, and improved performance by using single CNT transistors.

To conclude, the DNA-DNA detection demonstrated in this work can be extended to the detection of other biomolecules using artificial nucleic acids known as aptamers as probe molecules. These short (10-50 nucleotides) DNA-like sequences have been shown to bind to nucleosides, proteins, drugs, and cells [14], so that our system can be generalized to detect these as well. The protocols and detection principles are the same, which imparts versatility to our CNT transistor sensing array. We provide an example of how an aptamer detects protein in another paper in this issue. [15]

\section{ACKNOWLEDGMENT}

The authors thank Dr. J. S. Becker at Cambridge Nanotech, Inc., for providing the ALD coatings.

\section{REFERENCES}

[1] P. Bergveld, "Development, operation, and application of the ion-sensitive field effect transistor as a tool for electrophysiology," IEEE Trans. Biomed. Eng., vol. BME-19, pp. 342-351, Sep. 1972.

[2] E. Souteyrand, J. P. Cloarec, J. R. Martin, C. Wilson, I. Lawrence, S. Mikkelsen, and M. F. Lawrence, "Direct detection of the hybridization of synthetic homo-oligomer DNA sequences by field effect," J. Phys. Chem. B, vol. 101, no. 15, pp. 2980-2985, Apr. 1997.

[3] F. Pouthas, C. Gentil, D. Cote, G. Zeck, B. Straub, and U. Bockelmann, "Spatially resolved electronic detection of biopolymers," Phys. Rev. E, vol. 70, no. 3, pp. 031906-031906, Sep. 2004.

[4] F. K. Perkins, S. J. Fertig, K. A. Brown, D. McCarthy, L. M. Tender, and M. C. Peckerar, "An active microelectronic transducer for enabling label-free miniaturized chemical sensors," in Proc. IEEE Int. Electron Devives Meeting, IEDM Tech. Digest, Dec. 10-13, 2000, pp. 407-410.

[5] J. Fritz, E. B. Cooper, S. Gaudet, P. K. Sorger, and S. R. Manalis, "Electronic detection of DNA by its intrinsic molecular charge," PNAS, vol. 99, no. 22, pp. 14142-14146, Oct. 2002.

[6] F. Bendriaa, F. Le-Bihan, A. C. Salaun, T. Mohammed-Brahim, C. Tlili, N. Jaffrezic, and H. Korri-Youssoufi, "DNA detection by suspended gate polysilicon thin film transistor," IEEE Sensors, p. 4 pp., Oct. 30-Nov. 32005.
[7] J. Hahm and C. M. Lieber, "Direct ultrasensitive electric detection of DNA and DNA sequence variations using nanowire nanosensors," Nano Lett., vol. 4, no. 1, pp. 51-54, Jan. 2004.

[8] A. Star, E. Tu, J. Niemann, J. P. Gabriel, C. S. Joiner, and C. Valcke, "Label-free detection of DNA hybridization using carbon nanotube network field-effect transistors," PNAS, vol. 103, no. 4, pp. 921-926, Jan. 2006.

[9] H. Dai, "Nanotube growth and characterization," in Topics in Applied Physics. Heidelberg, Germany: Springer-Verlag, 2001, vol. 80, pp. 29-53.

[10] C. D. Fung, P. W. Cheung, and W. H. Ko, "A generalized theory of an electrolyte-insulator-semiconductor field-effect transistor," IEEE Trans. Electron Device, vol. ED-33, no. 1, pp. 8-18, Jan. 1986.

[11] J. B. Allen and R. F. Larry, Electrochemical Methods: Fundametals and Applications. New York: Wiley, 2000.

[12] R. Martel, V. Derycke, C. Lavoie, J. Appenzeller, K. K. Chan, J. Tersoff, and P. Avouris, "Ambipolar electrical transport in semiconducting single-wall carbon nanotubes," Phys. Rev. Lett., vol. 87, no. 25, pp. 256805-256805, Dec. 2001.

[13] W. Kim, A. Javey, O. Vermesh, Q. Wang, Y. Li, and H. Dai, "Hysteresis caused by water molecules in carbon nanotube field-effect transistors," Nano Lett., vol. 3, no. 2, pp. 193-198, Feb. 2003.

[14] A. D. Ellington and J. W. Szostak, "In vitro selection of RNA molecules that bind specific ligands," Nature, vol. 346, no. 6287, pp. 818-822, Aug. 1990.

[15] H. Pandana, K. H. Aschenbach, and R. D. Gomez, "Systematic aptamer-gold nanoparticle colorimetry for protein detection: Thrombin," IEEE Sensors J., vol. 8, no. 6, pp. 661-666, Jun. 2008.

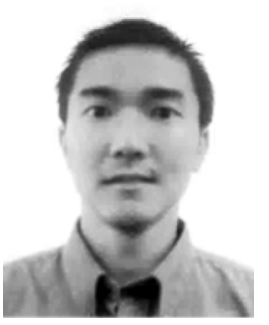

Herman Pandana was born in Medan, Indonesia He received the B.S. degree in electrical engineering from the National Taiwan University, Taipei, Taiwan, in 2000 . He is currently working towards the Ph.D. degree in biosensors at the Department of Electrical and Computer Engineering, University of Maryland, College Park.

His research interests include sensors for label-free biomolecule detection, semiconductor devices, and magnetic devices.

Mr. Pandana is a recipient of the Grant-in-Aid Award from the Cosmos Club, Washington, DC.

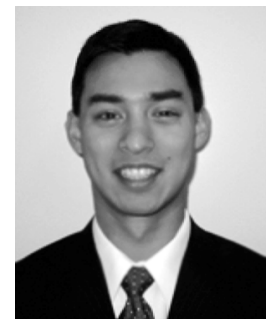

Konrad H. Aschenbach was born in Maryland. He received the B.S. degree (magna cum laude) in electrical engineering from the University of Maryland, College Park, in 2001 and the M.S. in electrical engineering from the University of California, Berkeley, in 2003. He is currently working towards the Ph.D. degree at the Department of Electrical and Computer Engineering, University of Maryland.

His current research interests are in the use of electronic sensors to detect biological molecules.

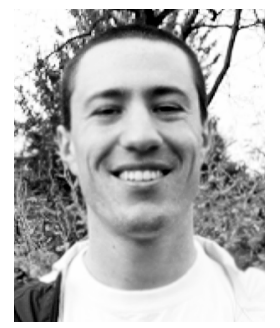

Daniel R. Lenski received the B.A. degree (cum laude) in physics and linguistics from Cornell University, Ithaca, NY, in 2003. He is currently working towards the Ph.D. degree at the Department of Physics, University of Maryland, College Park, focusing on the transport properties of organic semiconductors, and carbon nanotube-based devices. 


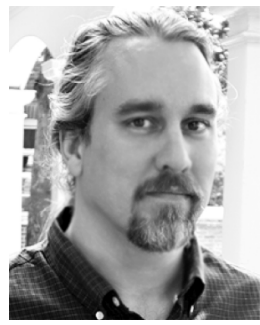

Michael S. Fuhrer received the B.S. degree from the University of Texas at Austin, Austin, and the Ph.D. degree from the University of California, Berkeley, in physics.

He is currently an Associate Professor of Physics at the University of Maryland, College Park. He has studied the first carbon nanotube heterojunctions, demonstrated the first carbon-nanotube-based single-electron memory device, and showed that the room-temperature mobility in semiconducting carbon nanotubes is the highest of any semiconductor. He has published over 35 papers in technical journals, and presented his research in more than 40 invited talks. He is pursuing research in nanoelectronics, studying electronic devices constructed from components that are naturally structured at the nanometer scale.

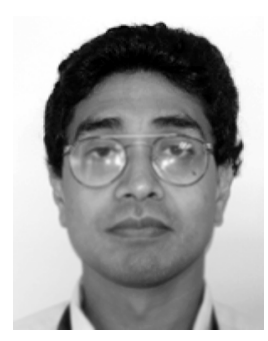

Javed Khan received the B.A. degree (Honors) in 1984, the M.B.B. Chir. (M.D.) degree in 1986, and M.A. degree in pathology, immunology and parasitology in 1989 all from Queens College, Cambridge University, Cambridge, U.K.

He became a member of the U.K. Royal College of Physicians in 1990 and received the U.S. General Pediatrics Board Certification in 2005. He joined the National Institutes of Health in 2001 and is currently a Principal Investigator and Attending Physician of the Pediatric Oncology Branch of the National Institutes of Health. His current research interests are in the area of microarrays and artificial intelligence for diagnosis and predicting prognosis in cancer patients. He has authored in excess of 40 scientific publications, including 11 review articles and coedited a book.

Dr. Khan is the recipient of multiple research related awards from the National Institutes of Health, the American Association for Cancer Research, the Children's Cancer Foundation, and the Hope Street Kids Foundation.

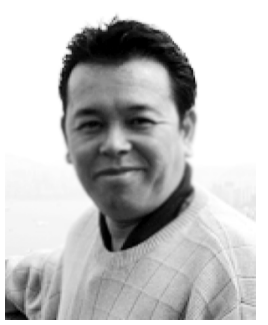

Romel D. Gomez (M'96-SM'05) was born in Manila, Philippines. He received the B.S. degree from the University of the Philippines, Manila, the M.S. degree from Wayne State University, Detroit, $\mathrm{MI}$, and the Ph.D. degree from the University of Maryland, College Park, all in physics.

He is currently a Professor of Electrical and Computer Engineering at the University of Maryland. He has authored more than 70 scientific publications, several book chapters, and holds two U.S. patents in magnetic imaging and scanned probe microscopy. His research interests are in the areas of magnetism, nanoelectronics, surface science, and biochemical sensors.

Dr. Gomez is the recipient of the U.S. National Science Foundation Career Award, as well as the George Corcoran, E. Robert Kent, and Keystone Professorial Awards of the Clark School of Engineering at the University of Maryland. He served as the General Chair of the IEEE Intermag 2006 Conference and the Chair of the Technical Committee of the IEEE Magnetics Society from 2004 to 2006. He was an Editorial Board Member of the IEEE TRANSACTIONS ON MAGNETICS from 2003 to 2005. 